\title{
Terahertz Compression of Electron Pulses at a Planar Mirror Membrane
}

\author{
Dominik Ehberger, ${ }^{1,2}$ Kathrin J. Mohler, ${ }^{1,2}$ Thomas Vasileiadis, ${ }^{4}$ Ralph Ernstorfer, ${ }^{4}$ Lutz Waldecker, ${ }^{5,4}$ \\ and Peter Baum ${ }^{1,2,3, *}$ \\ ${ }^{1}$ Ludwig-Maximilians-Universität München, Am Coulombwall 1, 85748 Garching, Germany \\ ${ }^{2}$ Max-Planck-Institute of Quantum Optics, Hans-Kopfermann-Str. 1, 85748 Garching, Germany \\ ${ }^{3}$ Fachbereich Physik, Universität Konstanz, Universitätsstr. 10, 78467 Konstanz, Germany \\ ${ }^{4}$ Fritz-Haber-Institut der Max-Planck-Gesellschaft, Faradayweg 4-6, 14195 Berlin, Germany \\ ${ }^{5}$ Department of Applied Physics, Stanford University, 348 Via Pueblo Mall, Stanford, CA 94305, USA
}

(Received 7 November 2018; revised manuscript received 13 December 2018; published 13 February 2019)

\begin{abstract}
Compression of electron pulses with terahertz radiation offers short pulse durations and intrinsic subcycle stability in time. We report the generation of 12-fs (rms), 28-fs (FWHM) electron pulses at a kinetic energy of $75 \mathrm{keV}$ by using single-cycle terahertz radiation and a simple planar mirror. The mirror interface provides transverse velocity matching and spatially uniform compression in time with purely longitudinal field-electron interaction. The measured short-term and long-term temporal drifts are substantially below the pulse duration without any active synchronization. A simple phase-space model explains the measured temporal focusing dynamics for different compressor strengths and shows a path toward generating isolated attosecond electron pulses from beams of almost arbitrary transverse emittance.
\end{abstract}

DOI: 10.1103/PhysRevApplied.11.024034

\section{INTRODUCTION}

Robust and reliable methods for generating femtosecond and attosecond electron pulses are key to studying atomic and electronic processes in matter on their fundamental length and time scales. Ultrafast electron microscopy and diffraction $[1,2]$ allow for observing ultrafast processes on femtosecond and subnanometer levels. The techniques have already elucidated numerous ultrafast solid-state phenomena on subpicosecond time scales [3-6], and subfemtosecond phenomena await investigation $[7,8]$. Proof-of-principle diffraction and microscopy with electron-pulse trains [9] indicate the practicability of this attosecond-Angstrom regime.

For pump-probe geometries with isolated pulses, time resolution is limited by the duration of the electron pulses and their stability in time with respect to the optical excitation. Recent approaches for electron-pulse compression and metrology use terahertz single-cycle pulses [10,11] instead of microwave radiation [12-15].

*peter.baum@lmu.de

Published by the American Physical Society under the terms of the Creative Commons Attribution 4.0 International license. Further distribution of this work must maintain attribution to the author(s) and the published article's title, journal citation, and DOI.
This concept largely circumvents temporal synchronization issues because an entire experiment can be operated in an all-optical way by a single laser source. In addition, terahertz radiation has been used for acceleration $[11,16]$, streaking and deflection $[10,11]$, focusing [11], and tilting [17] of electron pulses. There is also growing interest in applying terahertz techniques for the modulation of relativistic electron beams [18-20]. Waveguides or resonant structures are usually applied for mediating the terahertz-electron interaction. Although such structures can provide field enhancement and phase-matched propagation, their size limits the maximum acceptable electron-beam diameter. Therefore, it has been difficult to compress the rather large and highemittance electron beams of table-top experiments or linear electron accelerators down to few-femtoseconds duration.

We demonstrate the use of a simple planar membrane mirror in combination with a high-acceleration electron source for terahertz-based electron-pulse compression. The mirror provides transverse phase matching over an almost arbitrarily large electron beam size and the lasertriggered high-field electron source minimizes the effects of nonlinearities in the time domain. In the experiment, we compress few-electron pulses from a simple planar photocathode to durations of $12 \mathrm{fs}$ (rms) or $28 \mathrm{fs}$ (FWHM). These values are on par with state-of-the-art microwave compression techniques [15,21], but without the need for active synchronization $[22,23]$. 


\section{CONCEPT AND EXPERIMENTAL SETUP}

Figure 1(a) depicts the experimental setup. Electron pulses are emitted from a gold-coated photocathode by the second harmonic of 270-fs-long laser pulses from a Yb:YAG laser (Pharos, Light Conversion Inc.) at a repetition rate of $50 \mathrm{kHz}$. The electron source is of compact design, featuring a high electrostatic acceleration field close to the breakdown limit [24]. Carefully polished gun parts and a gap-free contact between the sapphire cathode and the metal construction ensure stable gun operation without field emission or arcing. The electron pulses are accelerated to a kinetic energy of $75 \mathrm{keV}$ over a distance of $11 \mathrm{~mm}$. The laser spot size on the cathode is roughly $5 \mu \mathrm{m}$ (FWHM). To avoid space-charge effects, we reduce the average number of emitted electrons per pulse to approximately 3 . The temporal shape of such a few-electrons pulse and its duration are understood as the probability distribution of the individual electron's arrival time at a certain position [25]. It is obtained from averaging over repetitive measurements. The electron-pulse duration at a distance of $0.9 \mathrm{~m}$ behind the gun is $490 \mathrm{fs}$ (FWHM), measured by terahertz streaking [10]. A magnetic solenoid lens is placed $12 \mathrm{~cm}$ after the gun exit and aligned collinearly to
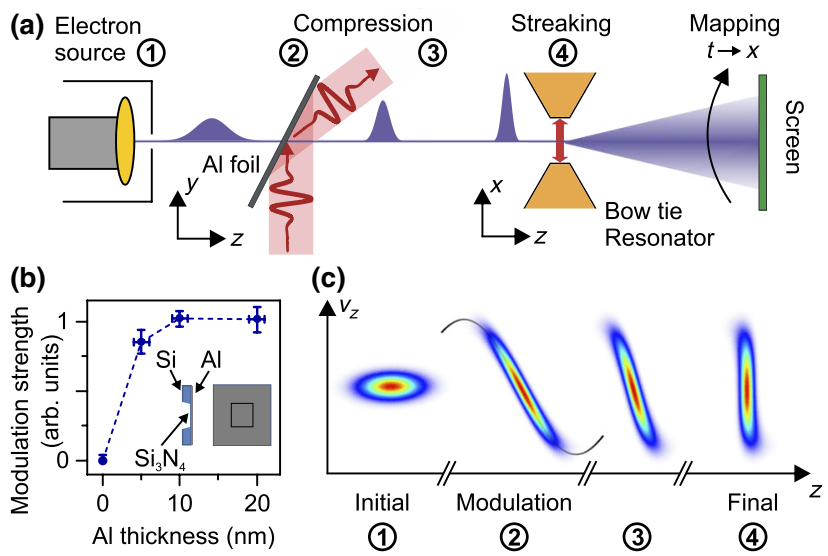

(c)

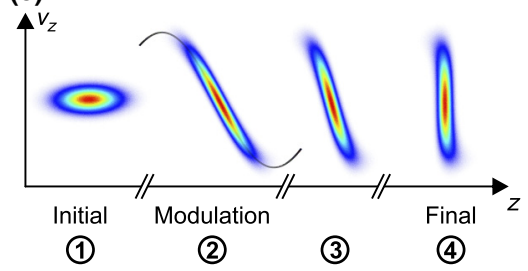

FIG. 1. Concept and experimental setup. (a) Schematic of the electron compression scheme based on an electron-transparent planar terahertz mirror. An initial electron pulse (1) emitted from the electron source propagates to the mirror, where it interacts with the terahertz wave (2) and compresses in time upon propagation (3). Streaking (4) allows for retrieval of the electron-pulse duration as it maps electron arrival times $(t)$ onto positions on the screen $(x)$. For the $50-\mu \mathrm{m}$ aperture (see text), the electron beam diameters at the compression mirror, bow-tie resonator, and screen are approximately $1 \mathrm{~mm},<30 \mu \mathrm{m}$, and approximately $150 \mu \mathrm{m}$, respectively. (b) Measured modulation strength at the terahertz compressor for different aluminum layer thicknesses. Inset: Schematic of the mirror element. (c) Phase space representation of the stages of electron-pulse compression (1-4) at terahertz frequencies. The black line indicates the terahertzinduced velocity gain along the electron pulse's longitudinal profile. the beam axis [26]. Terahertz single-cycle pulses for compression and subsequent streaking metrology are generated at a central frequency of $0.4 \mathrm{THz}$ in two separate $\mathrm{LiNbO}_{3}$ slabs with silicon prisms as output couplers [27]. Pump powers of approximately $3 \mathrm{~W}$ and $12 \mathrm{~W}$ are needed for compression and streaking, respectively.

Compression of the electron pulses is enabled by intersecting the electron beam with one of the two terahertz beams at approximately $90^{\circ}$ on an ultrathin aluminum mirror membrane located $0.4 \mathrm{~m}$ behind the electron source [see Fig. 1(a)]. The mirror is thin enough to transmit the electron beam, but thick enough to efficiently reflect the terahertz radiation. To find the best mirror for our experiment, we measure the amplitude of the terahertz-induced, time-dependent electron-pulse deflection of four different mirror membranes. Figure 1(b) depicts the relative strength of the interaction as a function of aluminum layer thickness. The strength of the deflection is proportional to the amount of longitudinal acceleration or deceleration [17]. We find that an aluminum layer as thin as $5-10 \mathrm{~nm}$ is sufficient, although it is $>10^{4}$ times thinner than a terahertz wavelength. This observation is in agreement with conductivity measurements of thin aluminum films at terahertz frequencies [28]. Simulations show that single atomic layers such as graphene might also be applicable [29]. In the experiment, the 10 -nm-thick aluminum layer is supported by a freestanding, 10 -nm-thick $\mathrm{Si}_{3} \mathrm{~N}_{4}$ membrane for mechanical stability [see inset of Fig. 1(b)]. The useful aperture is $1.5 \times 1.5 \mathrm{~mm}^{2}$ and the electron transmission for perpendicular incidence is approximately 0.7 .

At a distance of $0.5 \mathrm{~m}$ after the compression stage, the electron pulses are characterized in time by terahertz streaking [10] at a collinearly excited bow-tie-shaped terahertz resonator with a clear aperture of $80 \mu \mathrm{m}$. We use the magnetic lens to focus through the compression element and through the bow-tie resonator via an intermediate beam waist in front of the screen. There are no electrooptical elements between the compression and the streaking stage in order to avoid potential temporal distortions [30]. Different apertures of $25-50 \mu \mathrm{m}$ in diameter in front of the bow-tie resonator allow us to trade signal intensity for temporal-streaking resolution.

Figure 1(c) depicts the compression dynamics in longitudinal phase space. The trailing part of an initial electron pulse is accelerated and its leading part is decelerated, while the mean velocity is left unaltered. The initially trailing parts catch up with the previously leading parts of the pulse at a location of the shortest pulse duration called the temporal focus. If the incoming electron pulse is shorter than half a modulation cycle, we obtain a single, isolated time-compressed pulse [10]. Our planar mirror provides the required, time-dependent electron momentum modulation by letting the electrons abruptly enter the electromagnetic field within a time much shorter than a cycle period [31,32]. The final net momentum kick $\Delta p$ is given 
by the time-integrated Lorentz force acting on the particle until the terahertz field has passed. For a $p$-polarized plane wave of peak electric field strength $E_{0}$ and optical angular frequency $\omega$, an analytic derivation yields $\Delta p \propto E_{0} \sin (\omega t) / \omega$, where $t$ is the electron arrival time at the mirror relative to the crest of the wave; the proportionality constant depends on the electron and optical incidence angle [17]. A cosine-shaped terahertz pulse (carrier envelope phase equal to zero) is, therefore, required for efficient electron-pulse compression. It provides a sine-like momentum modulation that is approximately linear around its zero crossing [17]. To this end, we convert the sine-like terahertz pulses emerging from the $\mathrm{LiNbO}_{3}$ crystal (carrier envelope phase near $\pm \pi / 2$ ) into cosine-shaped pulses at the compression element by accurately adjusting the focusing conditions and in particular the Gouy phase. The size of the terahertz beam waist at the mirror is $2.8 \mathrm{~mm}$ (FWHM), which is considerably larger than the electron beam. The terahertz phase fronts are, therefore, approximately planar in the region overlapping with the electrons.

Realistic electron beams have a finite emittance and hence a finite beam diameter, for example, approximately $1 \mathrm{~mm}$ at the compressor stage in our experiment. It is, therefore, necessary to consider the compression dynamics as a function of the transverse coordinate. For planar elements, there is a certain combination of electron incidence angle $\theta_{e}$ and terahertz incidence angle $\theta_{\mathrm{THz}}$ for which momentum modulation and hence compression is independent of the spatial electron-beam profile. This configuration is called the (transverse) velocity-matching condition [32], namely, $c / \sin \theta_{\mathrm{THz}}=v_{e} / \sin \theta_{e}$, where $v_{e}$ is the electron velocity and $c$ is the speed of light [17]. At these angles, the phase velocity of the terahertz pulses matches the velocity of the electron incidence along the mirror surface. This velocity-matching condition coincides with the absence of time-dependent transverse momentum transfer, and thus the net modulation is purely longitudinal and spatially homogeneous [17]. The entire electron beam is, therefore, compressed into isolated, short, and nontilted electron pulses at the temporal focus. We note that a similar condition of spatially homogenous interaction cannot be found for sideways deflection because net transverse momentum changes are absent in a velocitymatched mirror configuration [17]. The planar membrane is, therefore, ideal for electron-pulse compression while a microstructured resonator is ideal for their temporal analysis.

\section{RESULTS ON PULSE COMPRESSION}

In the experiment, we first adjust the timing of electron and terahertz pulses at the compression and streaking stages to zero crossings of the modulation [10]. We then vary the field strength at the compression mirror until the shortest electron pulses are observed at the streaking stage.
Figure 2(a) depicts the measured streaking profiles as a function of compressor field strength. Temporal focusing is evident by the formation of a waist of the pulse duration for the optimum compression strength (peak field of approximately $3 \times 10^{5} \mathrm{~V} / \mathrm{m}$ ). We observe that the temporal pulse profile is different when the electron pulses are under compressed (relative field strength $<1$ ) as compared to over compression (relative field strength $>1$ ), where a doublepeak structure emerges. This effect has been observed before $[10,11]$ and is a direct consequence of the remaining temporal nonlinearities of the compression field [33]. At highest compression strength, these two peaks also have slightly different intensities because the curvature of the terahertz-modulation cycle is slightly different before and after the zero crossing. There is also a small background intensity for positive times in Fig. 2(a), which is reproducible and a consequence of electron energy loss due to inelastic scattering at the membrane mirror [34].

Figure 2(b) shows the results of a Monte Carlo simulation in the longitudinal domain [compare Fig. 1(c)] with $10^{7}$ point particles per simulated compression strength. Electron-electron interactions are neglected and
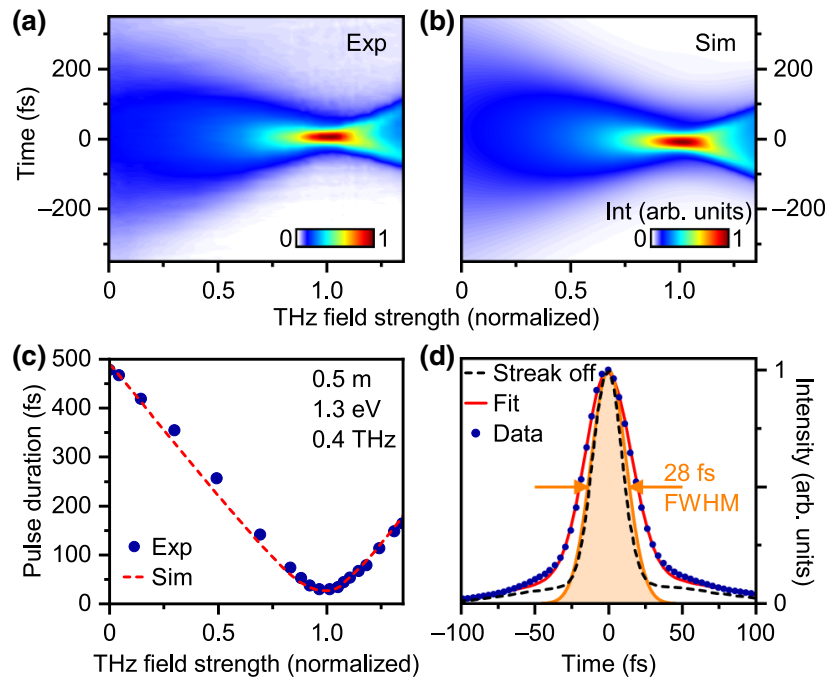

FIG. 2. Experimental results and simulation. (a) Measured streaking profiles as a function of the normalized compressor field strength (unity approximately corresponds to $3 \times$ $10^{5} \mathrm{~V} / \mathrm{m}$ ). The electron pulses first become shorter in time, reach a minimum pulse duration, and then become longer again for the highest field strengths. (b) Simulated streaking profiles assuming an energy spread of $1.3 \mathrm{eV}$, initial pulse duration of $490 \mathrm{fs}$, compression-streaking distance of $0.5 \mathrm{~m}$, modulation with $0.4 \mathrm{THz}$, and phase offset of $80 \mathrm{mrad}$. (c) Measured (blue dots) and simulated (dashed line) electron-pulse durations (FWHM). For visibility, only every other data point is shown. (d) Shortest measured electron pulse. The averaged streaking profile (blue dots) is fitted by convoluting the unstreaked profile (dashed line) with a Gaussian pulse. The best fit (red line) is obtained for a pulse duration of $28.3 \mathrm{fs}$ (FWHM) or $12.0 \mathrm{fs}$ (rms). 
terahertz-induced momentum transfer is much smaller than the central electron momentum. The initial electron pulse is assumed to be uncorrelated in energy and time, which neglects the dynamics of acceleration within the gun [35]. We find that an initial pulse duration of $490 \mathrm{fs}$ (FWHM), a modulation frequency of $0.4 \mathrm{THz}$, and an energy width of $1.3 \mathrm{eV}$ (FWHM) yield the best overall agreement with our measured data [compare with Fig. 2(a)]. The energy spread of $1.3 \mathrm{eV}$ may be caused by a work-function mismatch [36], inhomogeneous emission sites, inelastic scattering at the $\mathrm{Al} / \mathrm{SiN}$ membrane, or fluctuations of the acceleration voltage.

In Fig. 2(c), we plot the measured pulse duration (blue dots) with increasing compressor field strength, compared to the simulation results (red dashed line). As expected, the pulses first compress and later overcompress as a function of the applied field strength, which agrees very well with the simulated pulse durations. The electron pulses are shortened in time by a factor of approximately 17 . Figure 2(d) reports the electron-pulse shape and duration at the best compression strength. The blue dots show the measured streaking profile. It is narrow, but shows a nonGaussian shape and asymmetric wings. These wings are also present in the compressed but nonstreaked electronbeam profile (dashed line) and caused by elastic and inelastic electron scattering in the $\mathrm{Al}$ foil of the compressor; they can, in principle, be minimized by appropriate aperture and imaging settings. In general, the measured streaking profile is a convolution of the actual temporal profile of the electron pulses and the transverse beam profile. To retrieve the electron-pulse duration, we employ an evaluation that was also used by Maxson et al. [21]. We fit the data points (blue) with a convolution of an assumed Gaussian temporal shape for the electron pulses with the measured transverse electron-beam profile (black dashed line). The fitting curve [red line in Fig. 2(d)] closely matches the experiment (blue dots). The retrieved electron-pulse profile plotted in orange has a duration of $(28.3 \pm 0.5)$ fs (FWHM) or (12.0 \pm 0.2$)$ fs (rms). Alternatively, deconvolution using a Wiener filter yields approximately 25 fs (FWHM). A second experiment with a $25-\mu \mathrm{m}$ instead of a $50-\mu \mathrm{m}$ beamlimiting aperture in the streaking setup yields the exact same fit result of $12.0 \mathrm{fs}$ (rms). This observation indicates that the electron pulses are compressed independently of the beam size, as expected from the velocity-matched mirror configuration.

\section{TIMING STABILITY}

A central benefit of all-optical terahertz compression as compared to microwave approaches is the expected passive stability in time [10]. Here, we report a jitter and drift characterization over three typical time scales. Figure 3(a) shows the long-term drifts $\Delta T$ derived from repetitively acquired streaking traces and their center positions on the
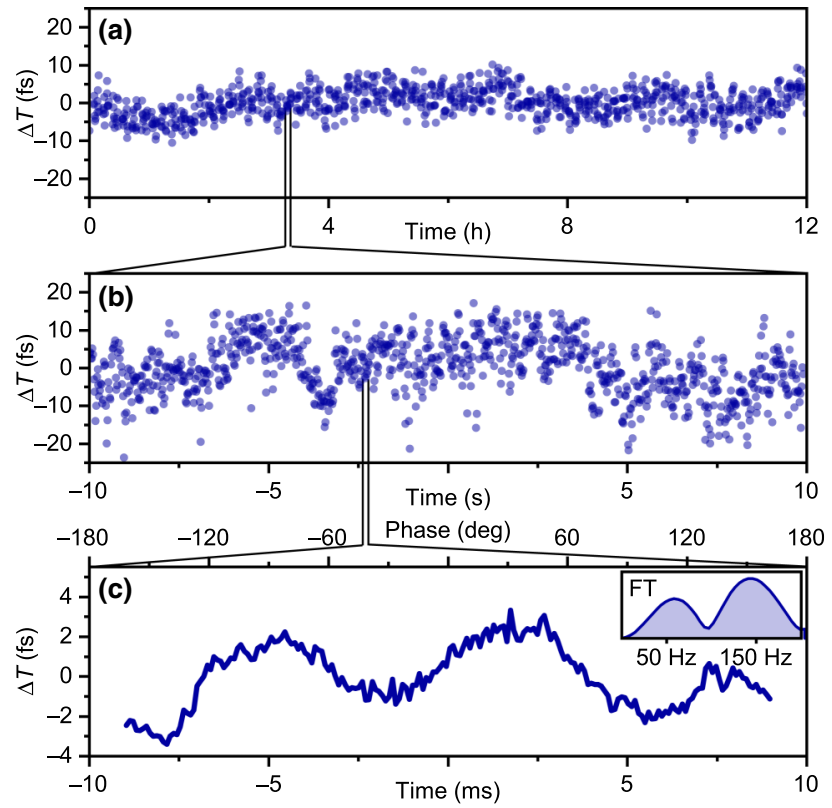

FIG. 3. Timing stability. (a) Long-term fluctuations amount to $3.7 \mathrm{fs}$ (rms). (b) Short-term jitter is $7.5 \mathrm{fs}$ (rms) on average. (c) Systematic timing error locked to $50 \mathrm{~Hz}$. Inset: Fourier analysis (FT).

screen for the best pulse-compression settings. Each data point is averaged for $20 \mathrm{~s}$. We measure a standard deviation of $3.7 \mathrm{fs}$ without observing systematic timing drifts over $12 \mathrm{~h}$. This result is obtained without employing any kind of active timing stabilization. Faster fluctuations are measured with the help of electronically driven capacitor plates behind the streaking resonator that deflect the electron beam perpendicularly to the streaking axis, that is, along the $y$ axis [see Fig. 1(a)]. This instrumentation allows us to measure timing fluctuations with millisecond resolution, limited mainly by the average electron current. Figure 3(b) shows a typical 20-s trace with each data point averaged over $21 \mathrm{~ms}$. The mean standard deviation of all 894 recorded 20 -s traces is 7.5 fs. The accumulated stability over the course of $12 \mathrm{~h}$ is $8.4 \mathrm{fs}$ (rms). Locking the capacitor plates to the $50-\mathrm{Hz}$ laboratory mains reveals systematic timing jitter on millisecond timescales. Figure 3(c) shows the result, averaged over approximately $10^{6} 50-\mathrm{Hz}$ cycles. We see a periodic modulation with an amplitude of $<2 \mathrm{fs}$ and with Fourier components at 50 and $150 \mathrm{~Hz}$ [Fig. 3(c), inset]. We attribute the remaining timing fluctuations to pointing variations of the terahertz-pump beams and/or high-voltage instabilities of the electron acceleration. All of the measured timing fluctuations are clearly below the pulse duration. The reported mirror-based terahertz compression concept thus enables low-dose, fewelectrons diffraction [37] and microscopy [1] at the highest temporal resolution, even for measuring times of several hours. 


\section{DISCUSSION}

The compressed electron pulses are 2-3 times shorter than those achieved with terahertz compression at bow-tie resonators [10] or other more elaborate interaction elements [11]. The advancement by a factor of $2-3$ over our previous results $[10,17]$ is a consequence of the dedicated mirror design with highest electron transmission and lowest electron energy losses combined with a substantially improved electron source [24].

The mirror compressor concept offers a clear perspective to enter the subfemtosecond regime with isolated electron pulses instead of pulse trains $[9,38,39]$. We first consider the potential influence of temporal nonlinearities of the compressing $\mathrm{THz}$ cycle. Figure 4(a) shows a simulation of compressed electron-pulse profiles obtained by the phasespace model when changing the electron-pulse durations at the compressor. Longer pulses result in stronger deviations from a Gaussian shape, caused by temporal nonlinearities of the terahertz modulation. Nevertheless, the FWHM pulse duration is hardly affected and only the peak electron current decreases while the background comes up. The final pulse duration in the modeled experimental geometry is rather dominated by the initial energy spread. Therefore, we next consider the influence of kinetic energy and bandwidth of the photoemission process on the compressed electron-pulse duration.

Figure 4(b) shows compressed pulse durations for different uncorrelated electron-energy spreads as a function of kinetic energy. We simulate two different compressorstreaking distances while keeping the initial pulse duration fixed to $490 \mathrm{fs}$. For a photocathode bandwidth of $0.5 \mathrm{eV}[36,40]$, the model predicts a final pulse duration of
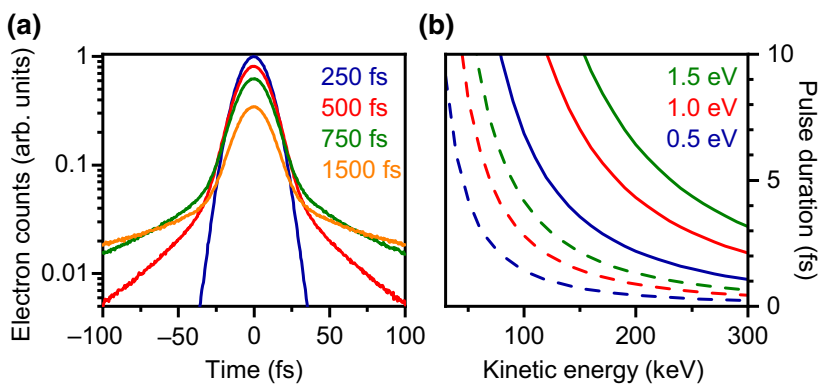

FIG. 4. Simulated electron-pulse shapes and durations. (a) Compressed electron-pulse profiles for different incoming electron-pulse durations (FWHM) at the compressor, assuming $0.4 \mathrm{THz}$ central modulation frequency. For longer electron pulses incident on the compression element, shoulders emerge in the temporal profile due to temporal nonlinearities of the modulation. (b) Compressed electron-pulse duration (FWHM) as a function of kinetic energy of the incoming electron pulses for different energy spreads. The initial electron-pulse duration is 490 fs. Solid lines, compression-streaking distance of $0.5 \mathrm{~m}$; dashed lines, compression-streaking distance of $0.1 \mathrm{~m}$. approximately 10 fs (FWHM) for the reported experimental configuration (blue solid line). Higher electron energies are also beneficial. Shortening the temporal focus distance from 50 to $10 \mathrm{~cm}$ at electron energies of $100-200 \mathrm{keV}$ breaks the 1-fs barrier. The required terahertz field strength on the order of $3 \times 10^{6} \mathrm{~V} / \mathrm{m}$ [17] for this case is realistically achievable [11]. We note that phase-space correlations due to acceleration in the electron gun are neglected in our model, and the above results are, therefore, the upper limits. In a multistage compressor as outlined in Ref. [10], the terahertz-driven planar mirror could serve to inject subcycle electron pulses into a higher-frequency compression stage $[9,38,39]$. The final pulse duration in such a multistage compressor is expected to be largely independent of residual jitter in the terahertz stage [39].

In summary, terahertz compression of electron pulses with the help of a planar mirror membrane is insensitive to electron-beam-size effects and provides a uniform, passively stable, and purely longitudinal momentum modulation. A state-of-the-art electron source minimizes temporal nonlinearities and reduces background in the temporal pulse profile. The generated electron pulses with their sub30-fs duration (FWHM) should be directly suitable for pump-probe investigations of fastest atomic and electron motion, for example, fundamental phonon modes [41,42] or metamaterial dynamics [43].

\section{ACKNOWLEDGMENTS}

This work was supported by the European Research Council (ERC) under the European Union's Horizon 2020 research and innovation program (Grant Agreements No. 647771 and No. ERC-2015-CoG-682843), the Munich Centre of Advanced Photonics (MAP), the International Max Planck Research School for Advanced Photon Science (IMPRS-APS) and the Max Planck Society. LW acknowledges support by the Alexander von Humboldt foundation. We would like to thank Ann-Kathrin Raab, Sonja Tauchert, Johannes Thurner, and Maxim Tsarev for assistance with electron beam-line components.

[1] A. H. Zewail, Four-dimensional electron microscopy, Science 328, 187 (2010).

[2] R. J. D. Miller, Femtosecond crystallography with ultrabright electrons and x-rays: Capturing chemistry in action, Science 343, 1108 (2014).

[3] B. J. Siwick, J. R. Dwyer, R. E. Jordan, and R. J. D. Miller, An atomic-level view of melting using femtosecond electron diffraction, Science 302, 1382 (2003).

[4] P. Baum, D. S. Yang, and A. H. Zewail, 4D visualization of transitional structures in phase transformations by electron diffraction, Science 318, 788 (2007).

[5] L. Waldecker, T. A. Miller, M. Rudé, R. Bertoni, J. Osmond, V. Pruneri, R. E. Simpson, R. Ernstorfer, and S. Wall, Time-domain separation of optical properties from 
structural transitions in resonantly bonded materials, Nat. Mater. 14, 991 (2015).

[6] T. Frigge, B. Hafke, T. Witte, B. Krenzer, C. Streubühr, A. S. Syed, V. M. Trontl, I. Avigo, P. Zhou, M. Ligges, D. von der Linde, U. Bovensiepen, M. Horn-von Hoegen, S. Wippermann, A. Lücke, S. Sanna, U. Gerstmann, and W. G. Schmidt, Optically excited structural transition in atomic wires on surfaces at the quantum limit, Nature 544, 207 (2017).

[7] H. C. Shao and A. F. Starace, Detecting Electron Motion in Atoms and Molecules, Phys. Rev. Lett. 105, 263201 (2010).

[8] V. S. Yakovlev, M. I. Stockman, F. Krausz, and P. Baum, Atomic-scale diffractive imaging of sub-cycle electron dynamics in condensed matter, Sci. Rep. 5, 14581 (2015).

[9] Y. Morimoto and P. Baum, Diffraction and microscopy with attosecond electron pulse trains, Nat. Phys. 14, 252 (2018).

[10] C. Kealhofer, W. Schneider, D. Ehberger, A. Ryabov, F. Krausz, and P. Baum, All-optical control and metrology of electron pulses, Science 352, 429 (2016).

[11] D. Zhang, A. Fallahi, M. Hemmer, X. Wu, M. Fakhari, Y. Hua, H. Cankaya, A.-L. Calendron, L. E. Zapata, N. H. Matlis, and F. X. Kärtner, Segmented terahertz electron accelerator and manipulator (STEAM), Nat. Photonics 12, 336 (2018).

[12] E. Fill, L. Veisz, A. Apolonski, and F. Krausz, Sub-fs electron pulses for ultrafast electron diffraction, New J. Phys. 8, 272 (2006).

[13] T. van Oudheusden, P. L. E. M. Pasmans, S. B. van der Geer, M. J. de Loos, M. J. van der Wiel, and O. J. Luiten, Compression of Subrelativistic Space-Charge-Dominated Electron Bunches for Single-Shot Femtosecond Electron Diffraction, Phys. Rev. Lett. 105, 264801 (2010).

[14] R. P. Chatelain, V. R. Morrison, C. Godbout, and B. J. Siwick, Ultrafast electron diffraction with radio-frequency compressed electron pulses, Appl. Phys. Lett. 101, 081901 (2012).

[15] A. Gliserin, M. Walbran, F. Krausz, and P. Baum, Subphonon-period compression of electron pulses for atomic diffraction, Nat. Commun. 6, 8723 (2015).

[16] E. A. Nanni, W. R. Huang, K.-H. Hong, K. Ravi, A. Fallahi, G. Moriena, R. J. D. Miller, and F. X. Kaertner, Terahertzdriven linear electron acceleration, Nat. Commun. 6, 8486 (2015).

[17] D. Ehberger, A. Ryabov, and P. Baum, Tilted Electron Pulses, Phys. Rev. Lett. 121, 094801 (2018).

[18] E. Curry, S. Fabbri, J. Maxson, P. Musumeci, and A. Gover, Meter-Scale Terahertz-Driven Acceleration of a Relativistic Beam, Phys. Rev. Lett. 120, 094801 (2018).

[19] L. Zhao, et al., Terahertz Streaking of Few-Femtosecond Relativistic Electron Beams, Phys. Rev. X 8, 021061 (2018).

[20] R. K. Li, M. C. Hoffmann, E. A. Nanni, S. H. Glenzer, M. E. Kozina, A. M. Lindenberg, B. K. Ofori-Okai, A. H. Reid, X. Shen, S. P. Weathersby, J. Yang, M. Zajac, and X. J. Wang, Terahertz-based subfemtosecond metrology of relativistic electron beams, Phys. Rev. Accel. Beams 22, 012803 (2019).

[21] J. Maxson, D. Cesar, G. Calmasini, A. Ody, P. Musumeci, and D. Alesini, Direct Measurement of Sub-10 fs Relativistic Electron Beams with Ultralow Emittance, Phys. Rev. Lett. 118, 154802 (2017).
[22] M. Walbran, A. Gliserin, K. Jung, J. Kim, and P. Baum, 5-Femtosecond Laser-Electron Synchronization for PumpProbe Crystallography and Diffraction, Phys. Rev. Appl. 4, 044013 (2015).

[23] M. R. Otto, L. P. René de Cotret, M. J. Stern, and B. J. Siwick, Solving the jitter problem in microwave compressed ultrafast electron diffraction instruments: Robust sub-50 fs cavity-laser phase stabilization, Struct. Dyn. 4, 051101 (2017).

[24] L. Waldecker, R. Bertoni, and R. Ernstorfer, Compact femtosecond electron diffractometer with $100 \mathrm{keV}$ electron bunches approaching the single-electron pulse duration limit, J. Appl. Phys. 117, 044903 (2015).

[25] P. Baum, On the physics of ultrashort single-electron pulses for time-resolved microscopy and diffraction, Chem. Phys. 423, 55 (2013).

[26] D. Kreier, D. Sabonis, and P. Baum, Alignment of magnetic solenoid lenses for minimizing temporal distortions, J. Opt. 16, 075201 (2014).

[27] M. V. Tsarev, D. Ehberger, and P. Baum, High-averagepower, intense $\mathrm{THz}$ pulses from a $\mathrm{LiNbO}_{3}$ slab with silicon output coupler, Appl. Phys. B 122, 30 (2016).

[28] N. Laman and D. Grischkowsky, Terahertz conductivity of thin metal films, Appl. Phys. Lett. 93, 051105 (2008).

[29] Y. Morimoto and P. Baum, Attosecond control of electron beams at dielectric and absorbing membranes, Phys. Rev. A 97, 033815 (2018).

[30] C. Weninger and P. Baum, Temporal distortions in magnetic lenses, Ultramicroscopy 113, 145 (2012).

[31] T. Plettner, R. L. Byer, E. Colby, B. Cowan, C. M. S. Sears, J. E. Spencer, and R. H. Siemann, Visible-Laser Acceleration of Relativistic Electrons in a Semi-Infinite Vacuum, Phys. Rev. Lett. 95, 134801 (2005).

[32] F. O. Kirchner, A. Gliserin, F. Krausz, and P. Baum, Laser streaking of free electrons at $25 \mathrm{keV}$, Nat. Photonics 8, 52 (2014).

[33] P. Baum and A. H. Zewail, Attosecond electron pulses for 4D diffraction and microscopy, Proc. Natl. Acad. Sci. U.S.A. 104, 18409 (2007).

[34] D. Ehberger, C. Kealhofer, and P. Baum, Electron energy analysis by phase-space shaping with $\mathrm{THz}$ field cycles, Struct. Dyn. 5, 044303 (2018).

[35] B. J. Siwick, J. R. Dwyer, R. E. Jordan, and R. J. D. Miller, Ultrafast electron optics: Propagation dynamics of femtosecond electron packets, J. Appl. Phys. 92, 1643 (2002).

[36] M. Aidelsburger, F. O. Kirchner, F. Krausz, and P. Baum, Single-electron pulses for ultrafast diffraction, Proc. Natl. Acad. Sci. U.S.A. 107, 19714 (2010).

[37] S. Lahme, C. Kealhofer, F. Krausz, and P. Baum, Femtosecond single-electron diffraction, Struct. Dyn. 1, 034303 (2014).

[38] K. E. Priebe, C. Rathje, S. V. Yalunin, T. Hohage, A. Feist, S. Schäfer, and C. Ropers, Attosecond electron pulse trains and quantum state reconstruction in ultrafast transmission electron microscopy, Nat. Photonics 11, 793 (2017).

[39] M. Kozák, N. Schönenberger, and P. Hommelhoff, Ponderomotive Generation and Detection of Attosecond FreeElectron Pulse Trains, Phys. Rev. Lett. 120, 103203 (2018). 
[40] D. Ehberger, J. Hammer, M. Eisele, M. Krüger, J. Noe, A. Högele, and P. Hommelhoff, Highly Coherent Electron Beam From a Laser-Triggered Tungsten Needle Tip, Phys. Rev. Lett. 114, 227601 (2015).

[41] K. Ishioka, M. Hase, M. Kitajima, L. Wirtz, A. Rubio, and H. Petek, Ultrafast electron-phonon decoupling in graphite, Phys. Rev. B 77, 121402 (2008).
[42] L. Waldecker, T. Vasileiadis, R. Bertoni, R. Ernstorfer, T. Zier, F. H. Valencia, M. E. Garcia, and E. S. Zijlstra, Coherent and incoherent structural dynamics in laser-excited antimony, Phys. Rev. B 95, 054302 (2017).

[43] A. Ryabov and P. Baum, Electron microscopy of electromagnetic waveforms, Science 353, 374 (2016). 